\title{
Attachment of Cells to Islands Presenting Gradients of Adhesion Ligands
}

\author{
Rafe T. Petty, Hung-Wing Li, Jane H. Maduram, Rustem Ismagilov, and Milan Mrksich* \\ Department of Chemistry and Howard Hughes Medical Institute, The University of Chicago, \\ Chicago, Illinois 60637
}

Received May 18, 2007; E-mail: mmrksich@uchicago.edu

\begin{abstract}
Mammalian and bacterial cells live in gradients. Soluble and immobilized gradients of signaling proteins guide the trafficking of cells and direct their development and maintenance. ${ }^{1}$ New methods to determine the influence of gradients in solution on cellular function have been critical to understanding chemotaxis, ${ }^{2 a}$ differentiation, ${ }^{2 \mathrm{~b}}$ and morphogenesis. ${ }^{2 \mathrm{c}}$ The development of strategies to prepare gradients of immobilized species, by contrast, has been more difficult and is still at an early stage. ${ }^{3}$ These strategies should combine surface chemistries to immobilize ligands in defined densities, orientations, and conformations and accommodate the preparation of surfaces that are geometrically patterned, for example, to prepare shapes for the attachment and spreading of individual cells. In this paper, we report a method that combines gradients of soluble ligands in microfluidic networks ( $\mu \mathrm{FNs})$ with immobilization chemistries on self-assembled monolayers (SAMs) to prepare well-defined gradients of adhesion molecules for the attachment of cells.
\end{abstract}

Several methods have been reported for the generation of biologically relevant, substrate-bound gradients including the electrochemical desorption of alkanethiolates from monolayers, ${ }^{4 a}$ photochemical patterning, ${ }^{4 \mathrm{~b}}$ diffusive patterning, ${ }^{4 \mathrm{c}}$ and the use of $\mu$ FNs. ${ }^{5}$ These methods have enabled studies of cell migration, adhesion, and polarity, but are limited in the ability to define, at the molecular level, the activities and densities of ligands at the surface. Photochemical methods have been applied to create structurally well-defined surfaces, but are not compatible with all immobilization chemistries, including thiol addition to maleimide groups used in this work. ${ }^{6}$

In the current work we use $\mu$ FNs to establish a soluble gradient of the peptide GRGDSC (the "RGD" peptide) in contact with a monolayer that presents maleimide groups among tri(ethylene glycol) groups. ${ }^{7}$ In this way, the peptides are presented uniformly and at defined density. The RGD peptide is a ligand for cell-surface integrin receptors, which are used by most cells to attach to and sense the extracellular environment and to establish a cytoskeleton. ${ }^{8}$ The glycol groups prevent nonspecific interactions of proteins and cells with the surface. ${ }^{9}$ We demonstrate the preparation of approximately square features that present defined gradients to individual cells and show that the gradient of adhesion ligand leads to an nonuniform distribution of the cytoskeletal elements in adherent cells.

We used the $\mu \mathrm{FN}$ to combine a stream containing the RGD peptide with a stream containing the inactive GGRDGSC (the "RDG") peptide at the same concentration. ${ }^{10}$ The two peptides mix by diffusion to establish the gradient but maintain a uniform concentration of total peptide across the channel, ensuring that the immobilization reaction proceeds at a uniform rate and the immobilized gradient reflects the gradient of the RGD peptide in the $\mu \mathrm{FN}$. To pattern a monolayer with an array of squares (measuring $33 \mu \mathrm{m}$ ) each having a gradient of RGD peptide, we first used a serpentine microfluidic channel to block the maleimide

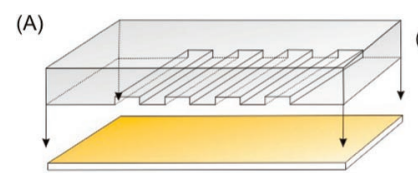

(B)

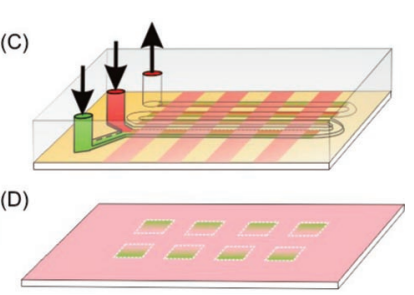

Figure 1. Scheme for preparing isolated gradient microislands. See the text for an explanation of each step.

groups with the inactive peptide RDG (Figure 1B). We then removed the microfluidic stamp and applied a Y-channel stamp that was used to flow a gradient of RGD peptide across the reactive stripes on the substrate (Figure 1C). In this way, individual gradients were created which were bounded by both sides of the microfluidic channel and by the presence of the blocked regions of the substrate from the first patterning step. We then removed the microfluidic device and immersed the entire surface in a solution of nonadhesive peptide to block the remaining maleimide groups and to give an array of isolated gradients of adhesive peptide (Figure 1D).

This sequential patterning scheme requires that immobilization of the peptides at distinct regions of the monolayer proceeds in high yield. To verify the reactions, we used SAMDI mass spectrometry to analyze the composition of a surface patterned with $\mu$ FNs. ${ }^{11}$ In this experiment, a $500 \mu \mathrm{m}$ wide stripe of RGD was patterned on the surface. Subsequently, the entire surface was immersed in a solution of the RDG peptide. SAMDI analysis of each region showed that the first patterning reaction was complete, and no subsequent immobilization of RDG was observed in the patterned region (Figure 2).

We next report studies of cell adhesion to the micropatterned gradients. We note that our method generates a gradient of gradients; that is, the gradient of active peptide becomes more diffuse as the fluid moves through the channel, yielding a continuum of immobilized gradients. This property can be useful for experiments that survey the dependence of a cellular function on geometry of the gradient. Importantly, analytical solutions exist for defining the gradient of peptide as a function of initial peptide concentrations, channel geometry, and flow rate ${ }^{5}$ and that account for the transverse diffusive broadening in laminar flows. ${ }^{12}$ The Supporting Information shows images of the gradients.

We placed the patterned substrates in a suspension of B16F10 mouse melanoma cells. The cells attached efficiently, with individual cells spreading within the gradient microislands (Figures 3). Prior examples have used surfaces that were modified with gradients at length scales larger than that of the cell and therefore focused on cell migration. ${ }^{\text {a }}$ Our use of islands that restrict individual cells allows a determination of the influence of a gradient of adhesion ligand on polarization of the cytoskeleton. Importantly, the microisland gradients preclude cell migration, which can also contribute to cell polarity. ${ }^{13}$ We fixed and immunostained the cells 


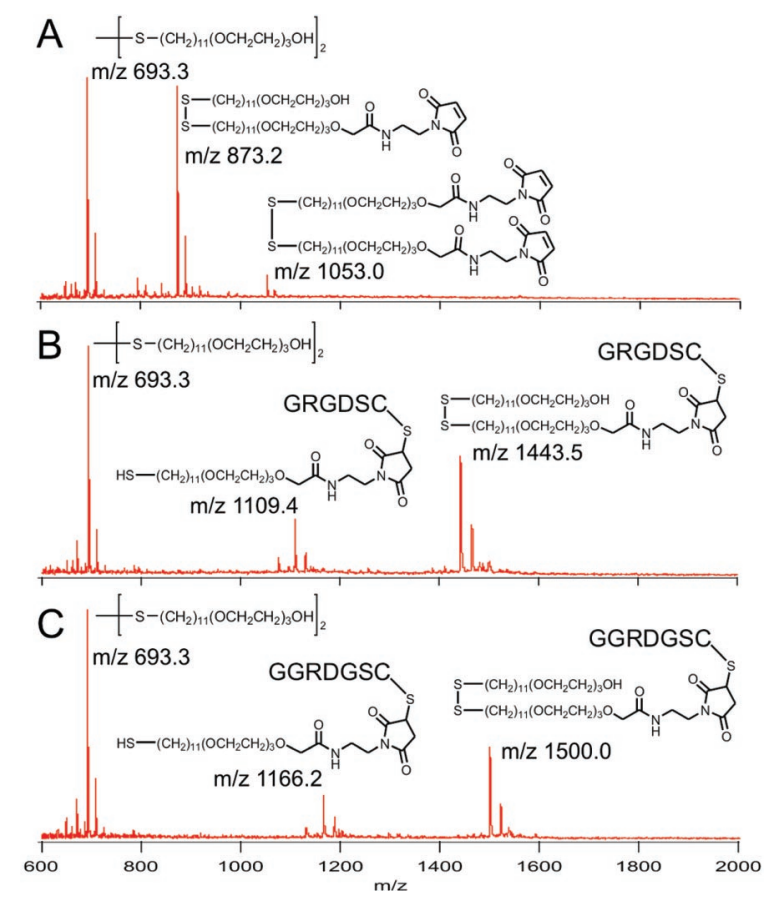

Figure 2. SAMDI-MS of the maleimide-terminated SAM (A) before and after reaction with the (B) RGD peptide and (C) RDG peptide.

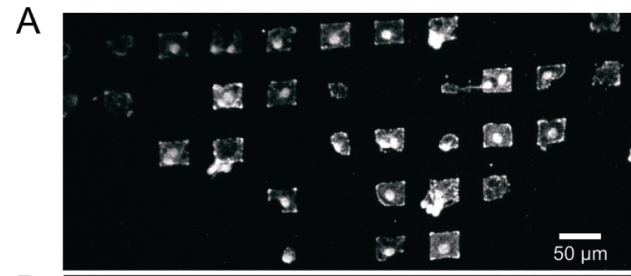

B

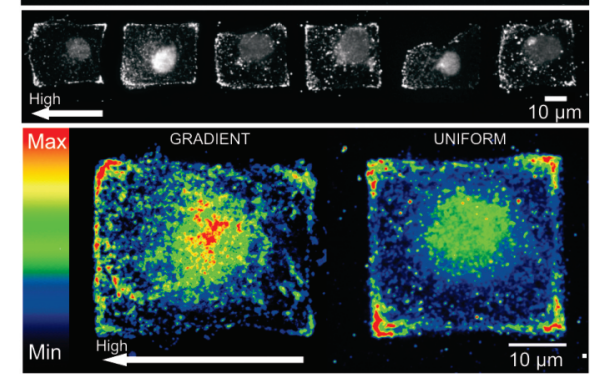

$\mathrm{D}$

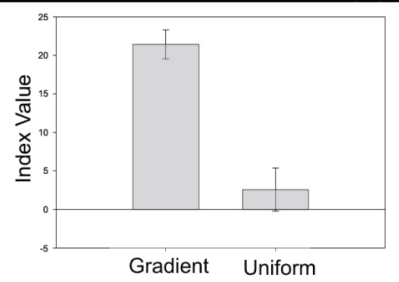

Figure 3. Representative views of B16/F10 melanoma cells stained for vinculin: (A) fluorescent image of fixed and immunostained cells on an array of gradient microislands; (B) representative images of single cells isolated on gradient islands; (C) representative heat maps of cells on gradient (left) and uniform (right) microislands. High RGD concentration is located on the left side of the gradient heat map. The total area of the islands is $\sim 1000 \mu \mathrm{m}^{2}$ (gradient, $n=22$; uniform, $n=11$ ); (D) average index values for four separate experiments. Error bars represent the standard error of measurement.

with an antibody against vinculin, which is found in the focal adhesion structures that integrate the cytoskeleton with the extracellular matrix. ${ }^{15} \mathrm{~A}$ visual inspection of the cells revealed a polarized FA distribution, with FAs preferentially located at regions of the substrate presenting a higher density of RGD.
Vinculin heat maps from independent experiments were generated by aligning and stacking images of individual cells to obtain the integrated pixel intensity. We imaged cells that were on gradient islands having a maximum slope (at the midpoint of the channel) of approximately $1.0 \times 10^{-12}$ to $7.6 \times 10^{-12} \mathrm{nmol} \mathrm{RGD} / \mu \mathrm{m}^{3}$. To quantitate the distribution of FAs on the high and low-density edges of the cell, we integrated the pixel density $(D$, reflective of the density of FA in the cell) on each edge of the cell (using a $\sim 5 \mu \mathrm{m}$ stripe $)$ and determined a polarity index as follows: $I=\left(D_{\mathrm{HIGH}}-\right.$ $\left.D_{\text {LOW }}\right) /\left(D_{\text {HIGH }}+D_{\text {LOW }}\right)$. The average values of the index for four independent experiments gave a mean index of $21 \pm 2$ (relative to a mean index of $2 \pm 2$ for cells on islands of uniform density) and clearly showed that the gradient of adhesive ligand influenced the cytoskeletal structure.

This Communication demonstrates a flexible and straightforward method for generating nonuniform patterns of substrate bound ligands on the length scale of single cells. We have shown that the resulting monolayers are compatible with studies of adherent cell culture and that cells exhibit a nonuniform distribution of FAs in response to a gradient of peptide adhesion ligand. This technique offers significant benefits in preparing substrates having welldefined gradients and patterns of immobilized ligands. We believe these tools will be especially important in understanding the influence of nonuniform microenvironments on cell function, including polarization and migration.

Acknowledgment. This work was supported by NSF-MRSEC and NIH. R.T.P. was supported by the NIH Chemistry \& Biology Interface Pre-Doctoral Training Program (Grant T32 GM008720).

Supporting Information Available: Details for fabrication of $\mu \mathrm{FNs}$, preparation of SAMs, cell culture, mass spectrometric analysis, fluorescent images of the gradient, and data analysis. This material is available free of charge via the Internet at http://pubs.acs.org.

\section{References}

(1) (a) Van Haastert, P. J. M.; Devreotes, P. N. Nat. Rev. Mol. Cell Biol. 2004, 5, 626-634. (b) Gurdon, J. B.; Bourillot, P.-Y. Nature 2001, 413 797-803

(2) (a) Mao, H.; Cremer, P. S.; Manson, M. D. Proc. Natl. Acad. Sci. U.S.A 2003, 100, 5449-5454. (b) Chung, B. G.; Flanagan, L. A.; Rhee, S. W. Schwartz, P. H.; Lee, A. P.; Monuki, E. S.; Jeon, N. L. Lab Chip 2005 5, 401-406. (c) Lucchetta, E. M.; Lee, J. H.; Fu, L. A.; Patel, N. H Ismagilov, R. F. Nature 2005, 434, 1134-1138

(3) Sniadecki, N. J.; Desai, R. A.; Ruiz, S. A.; Chen, C. S. Ann. Biomed Eng. 2006, 34, 59-74

(4) (a) Plummer, S. T.; Wang, Q.; Bohn, P. W. Langmuir 2003, 19, 75287536. (b) Ryan, D.; Parviz, B. A.; Linder, V.; Semetey, V.; Su, J.; Mrksich, M.; Whitesides, G. M. Langmuir 2004, 20, 9080-9088. (c) Smith, J. T.; Tomfohr, J. K.; Wells, M. C.; Beebe, T. P., Jr.; Kepler, T. B.; Reichert W. M. Langmuir 2004, 20, 8279-8286.

(5) (a) Jiang, X.; Xu, Q.; Dertinger, S. K. W.; Stroock, A. D.; Fu., T.-M Whitesides, G. M. Anal. Chem. 2005, 77, 2338-2347. (b) Fosser, K. A. Nuzzo, R. G. Anal. Chem. 2003, 75, 5775-5782. (c) Jeon, N. J.; Dertinger, S. K. W.; Chiu, D. T.; Choi, I. S.; Stroock, A. D.; Whitesides, G. M. Langmuir 2000, 16, 8311-8316. (d) Dertinger, S. K. W.; Jiang, X.; Li, Z.; Murthy, V. N.; Whitesides, G. M. Proc. Natl. Acad. Sci. U.S.A. 2002 $99,12542-12547$

(6) Dillmore, W. S.; Yousaf, M. N.; Mrksich, M. Langmuir 2004, 20, 72237231.

(7) Houseman, B. T.; Gawalt, E. S.; Mrksich, M. Langmuir 2003, 19, 15221531.

(8) Geiger, B.; Bershadsky, A.; Pankov, R.; Yamada, K. M. Nat. Rev. Mol. Cell Biol. 2001, 2, 793-805

(9) (a) Roberts, C.; Chen, C. S.; Mrksich, M.; Martichonok, V.; Ingber, D. E.; Whitesides, G. M. J. Am. Chem. Soc. 1998, 120, 6548-6555. (b) Houseman, B. T.; Mrksich, M. Biomaterials 2001, 22, 943-955.

(10) Giancotti, F. G. Curr. Opin. Cell. Biol. 1997, 9, 691-700.

(11) Su, J.; Mrksich, M. Langmuir 2003, 19, 4867-4870.

(12) Ismagilov, R. F.; Stroock, A. D.; Kenis, P. J. A.; Whitesides, G.; Stone, H. A. Appl. Phys. Lett. 2000, 76, 2376-2378.

(13) Huttenlocher, A. Nat. Cell. Bio. 2005, 7, 336-337.

(14) Ziegler, W. H.; Liddington, R. C.; Critchley, D. R. Trends Cell Biol. 2006, $16,453-460$

JA0735709 